\title{
Desenlaces críticos en pacientes pediátricos con hipertensión pulmonar y cardiopatía congénita llevados a cateterismo cardíaco diagnóstico en el Hospital Cardiovascular de Soacha entre 2013 y 2016
}

Jorge Mario Córdobaㅜ; Alfonso Velandia; Jimmy Andrés Riaño Cuastumal²; María Alejandra Ramírez²; William Montenegro ${ }^{3}$; María Camila Mejía ${ }^{*}$

1 Profesor Asociado Departamento de Anestesiología Fundación Universitaria de Ciencias de la Salud - Hospital de San José de Bogotá.

2 Residente de Anestesia Cardiovascular. Fundación Universitaria de Ciencias de la Salud - Hospital de San José de Bogotá.

3 Médico general, CJNC.

4 Médico general, Residente de Epidemiología, FUJNC.

Correspondencia: María Camila Mejía, e-mail: maria_camila_mg@hotmail.com

Como citar: Córdoba JM, Velandia A, Riaño JA, Ramírez MA, Montenegro W, Mejía MA. Desenlaces críticos en pacientes pediátricos con hipertensión pulmonar y cardiopatía congénita llevados a cateterismo cardíaco diagnóstico en el Hospital Cardiovascular de Soacha entre 2013 y 2016. Cuarzo 2015; 21 (2): 81-87.

Recibido: 20 de junio de 2015

Aceptado: 10 de octubre de 2015

Publicado: 15 de diciembre de 2015

Licencia creative commons

\section{Resumen}

INTRODUCCIÓN: Por cada mil nacidos vivos en Colombia, de dos a tres presentan algún tipo de anomalía congénita cardíaca; la mayoría de ellas estará asociada al desarrollo de hipertensión pulmonar, la cual es una condición fisiopatológica que según el grado de severidad afecta la morbilidad y la mortalidad, siendo un factor determinante para la operabilidad y pronóstico del paciente. El método diagnóstico definitivo es el cateterismo cardiaco procedimiento que permite evaluar la anatomía, el estado hemodinámico, la respuesta a los vasodilatadores pulmonares específicos y el grado de severidad de la condición, así como apoyar en la decisión del manejo quirúrgico definitivo; este procedimiento requiere en la mayoría de casos anestesia general. Sin embargo, el cateterismo no se encuentra exento de riesgos; se ha reportado una frecuencia de 4.5 - 5.7\% de paro cardíaco, un riesgo de muerte entre el $0.3-0.8 \%$, entre otras complicaciones. Por lo tanto el objetivo de este estudio fue evaluar la incidencia de complicaciones del cateterismo cardíaco en pacientes con cardiopatía congénita asociada a hipertensión pulmonar.

MÉTODOS: Se realizó un estudio de corte transversal en pacientes pediátricos con cardiopatía congénita asociada a hipertensión pulmonar llevados a cateterismo cardíaco diagnóstico bajo anestesia general en el periodo comprendido entre 2013 y 2016 en salas de hemodinamia del Hospital cardiovascular del niño de Cundinamarca. Se evaluó la presentación de complicaciones intraoperatorias, así como las características demográficas y clínicas de los pacientes antes del procedimiento. 
RESULTADOS: Se incluyeron 81 pacientes. La incidencia de complicaciones intraoperatorias durante el procedimiento fue para crisis de hipertensión pulmonar el 9\%, el 6\% de los pacientes presentaron choque intraoperatorio, 10\%, hipoxemia, el 4\% presentó algún tipo de arritmia colapsante y el 14\% requirió de ventilación mecánica prolongada, además se observó una mortalidad del 11\% dentro de los siguientes 30 días de hospitalización.

CONCLUSIONES: El cateterismo cardíaco en pacientes con anomalías congénitas es un procedimiento riesgoso que merece especial atención por parte del anestesiólogo con la finalidad de mitigar el riesgo de posibles complicaciones.

PALABRAS CLAVE: anestesia general, cardiopatías congénitas, diagnóstico, cateterismo cardíaco, hipertensión pulmonar.

\begin{abstract}
Critical outcomes in pediatric patients with pulmonary hypertension and congenital cardiopathy carried out to diagnostic heart catheterization in the Cardiovascular Hospital of Soacha between 2013 and 2016
\end{abstract}

BACKGROUND: For every thousand live births in Colombia, two to three present some type of cardiac congenital anomaly; the majority of them will be associated with the development of pulmonary hypertension, which is a pathophysiological condition that according to severity affects morbidity and mortality, being a determining factor for the operability and prognosis of the patient. The definitive diagnostic method is the cardiac catheterization procedure that allows to evaluate the anatomy, the hemodynamic state, the response to the specific pulmonary vasodilators and the degree of severity of the condition, as well as to support in the decision of the definitive surgical management; this procedure requires in most cases general anesthesia. However, catheterization is not without risk; it has been reported a frequency of 4.5 - 5.7\% of cardiac arrest, a risk of death between $0.3-0.8 \%$, among other complications. Therefore, the objective of this study was to evaluate the incidence of complications of cardiac catheterization in patients with congenital heart disease associated with pulmonary hypertension.

METHODS: A cross-sectional study was carried out in pediatric patients with congenital heart disease associated with pulmonary hypertension undergoing cardiac catheterization under general anesthesia was performed between 2013 and 2016 in hemodynamic rooms at the Cundinamarca Children's Hospital. We evaluated the presentation of intraoperative complications as well as the demographic and clinical characteristics of the patients before the procedure.

RESULTS: We included 81 patients. The incidence of intraoperative complications during the procedure was $9 \%$ for pulmonary hypertension, $6 \%$ of patients had intraoperative shock, $10 \%$ had hypoxemia, $4 \%$ presented some type of collapsing arrhythmia and $14 \%$ required mechanical ventilation In addition, a mortality rate of $11 \%$ was observed within the next 30 days of hospitalization. Conclusions: Cardiac catheterization in patients with congenital anomalies is a risky procedure that deserves special attention on the part of the anesthesiologist in order to mitigate the risk of possible complications.

KEYWORDS: anesthesia, general, heart defects, congenital, diagnosis, catheterization. hypertension, pulmonary.

\section{Introducción}

Es conocido que las cardiopatías congénitas se asocian frecuentemente a hipertensión pulmonar (HTP) y a un corto circuito sanguíneo que conlleva a un hiperflujo pulmonar constante, el cual de no ser corregido a tiempo se asociará con cambios estructurales de la vasculatura pulmonar de tipo irreversible; definida como una presión arterial media pulmonar $>25 \mathrm{~mm}$ Hg (mPAP), PAWP (presión en cuña) $<15$ mm Hg y PVRI (índice de resistencia) $>2 \mathrm{WU} / \mathrm{m} 2$ (1-3). El mecanismo fisiopatológico de la HTP desencadena remodelación vascular y disfunción endotelial con un proceso de vasoconstricción, inflamación, trombosis, proliferación, apoptosis celular y finalmente fibrosis, con aumento de la pos carga del ventrículo derecho y cambios en su estructura que finalmente producen 
deterioro de su función a etapas tan avanzadas que provocan la muerte. Por lo anterior, la mayoría de pacientes con esta condición fisiopatológica requieren de estudios invasivos como el cateterismo cardiaco con anestesia general, procedimiento necesario a la hora de toma de decisiones en la terapéutica farmacológica como en conducta quirúrgica del paciente.

Sin embargo, este procedimiento no se encuentra exento de riesgo, los reportes de literatura muestran entre el $4.5-5.7 \%$ de frecuencia de paro cardíaco y un riesgo de muerte que oscila entre el $0.3-0.8 \%$, todos asociados a crisis de hipertensión pulmonar y a arritmias colapsantes (2). Por tal motivo, este procedimiento representa un desafío para el anestesiólogo cardiovascular, quien debe hacerle frente a las situaciones que se presenten durante el cateterismo; es así que la valoración pre anestésica toma un gran valor para determinar probables factores que puedan verse relacionados con las complicaciones durante el periperatorio como puede ser el grado de hipertensión pulmonar, la edad del paciente, su peso, el consumo de medicación vasodilatadora o la asociación de variosde ellos. No obstante, durante el procedimiento, se modifican ciertas variables hemodinámicas que derivan en un cambio en la probabilidad de presentar complicaciones durante y después del procedimiento (2).

Existe poca información epidemiológica local acerca de la incidencia de las complicaciones en estos pacientes a pesar de que con frecuencia los pacientes pediátricos con cardiopatía congénita son sometidos a cateterismo cardíaco diagnóstico(4). Además de forma general, es muy improbable contar con recomendaciones de primer orden en estos tópicos por la escasez de estudios clínicos controlados a nivel internacional y estudios en Colombia, donde ciertas condiciones sociales, del paciente y de la atención modifican el riesgo. Por lo anterior es válido y relevante describir la experiencia de un centro de referencia nacional como es el Hospital Cardiovascular del Niño de Cundinamarca para generar hipótesis y en el futuro nuevos estudios de un mayor nivel de evidencia. El objetivo del presente estudio fue caracterizar la población de pacientes con anomalías congénitas asociadas a hipertensión pulmonar llevados a cateterismos diagnóstico y evaluar la incidencia de complicaciones durante el procedimiento.

\section{Metodología}

Se llevó a cabo un estudio descriptivo tipo corte transversal en el Hospital Cardiovascular del Niño de Cundinamarca. Se incluyó toda la población pediátrica entre 0 - 18 años con diagnóstico de alguna cardiopatía congénita asociado a hipertensión pulmonar que fuera sometida a cateterismo cardiaco diagnóstico durante el periodo comprendido entre 2013 a 2016. Se excluyeron los pacientes en quienes no fue posible documentar de manera suficiente el diagnóstico de hipertensión pulmonar por ecocardiograma. Se diseñó un formato de recolección de datos el cual consideraba variables relacionadas con las características sociodemográficas, clínicas, paraclínicas así como la presentación de desenlaces críticos durante y posterior al procedimiento. Para el estudio se definieron los desenlaces críticos intraoperatorios como se expresa a continuación: Crisis hipertensiva pulmonar intraoperatoria (Colapso hemodinámico relacionado a un incremento agudo en la resistencia vascular pulmonar definido a partir en un incremento en la presión arterial pulmonar acompañado por la disminución de la saturación de oxigeno sistémico y presión arterial sistémica); choque intraoperatorio (PAS < $90 \mathrm{mmHg}$ por más de 30 minutos o necesidad de aplicación de vasopresores); hipoxemia intraoperatoria (Descenso del 10\% del valor de SatO2 con respecto al valor previo al procedimiento); arritmia colapsante (cambio en el ritmo cardíaco que conlleva al colapso cardiovascular); requerimiento de cirugía urgente (necesidad de intervención quirúrgica de cualquier tipo durante el cateterismo); muerte intraoperatoria (muerte durante el cateterismo). De otro lado, los desenlaces posoperatorios tenidos en cuenta fueron: ventilación mecánica prolongada (Uso de ventilación mecánica $>48$ horas posterior al procedimiento); estancia prolongada en UCI (Estancia en la UCI > 48 horas posterior al procedimiento); muerte intrahospitalaria (muerte durante la estancia hospitalaria hasta 30 días posterior al procedimiento).

La información fue extraída a partir de lo consignado en la historia clínica electrónica con sistema operativo Healthycloud de la institución. El trabajo fue aprobado por el comité de ética de investigación del Hospital Cardiovascular del Niño de Cundinamarca. Los datos fueron analizados utilizando el programa estadístico STATA 13 (StataCorp. College Station, Texas 77845 USA). Se presentaron utilizando medidas de frecuencia (proporciones) y de tendencia central y dispersión de acuerdo al tipo de variable. 


\section{Resultados}

Durante el período de estudio, 120 pacientes fueron sometidos al procedimiento de cateterismo diagnostico por anomalía congénita. Se incluyeron 81 pacientes que cumplieron los criterios de elegibilidad pre-establecidos, mientras que 39 pacientes fueron excluidos de análisis debido a que no se contó con la información suficiente para determinar el diagnóstico de hipertensión pulmonar.

El 82.7\% de los pacientes tuvo reporte de pertenecer al régimen subsidiado. La mayoría fueron hombres (51\% Vs $49 \%)$. En relación a la edad, la mayoría de pacientes se encontraban en rango de 1 a 18 años (64.2 \%), no obstante, se analizaron datos de pacientes entre un mes y un año (30.9\%) y 0 a 1 mes de edad (4,9\%). El 12\% de la población fue prematura (Tabla 1). El tipo de anomalía cardíaca congénita más frecuente fue la comunicación interventricular (24.6\%), seguida por comunicación interauricular (23.4\%) y el drenaje venoso anómalo con el $13.5 \%$ (Tabla 2).

En relación al estado clínico del paciente durante la valoración preanestésica, se encontró que el 58\% presentaron cianosis, el $28 \%$ usaba oxígeno domiciliario y el $3,7 \%$ tuvo antecedente de insuficiencia renal crónica. De acuerdo al reporte del ecocardiograma realizado previo al cateterismo cardíaco, el $60.5 \%$ de los pacientes presentaron hallazgo de dilatación de cavidades derechas y un $27.5 \%$ mostró hipertrofia del ventrículo derecho. El grado de la hipertensión pulmonar medido por ecocardiograma clasificó a los pacientes como severa (73.7\%), moderada (23.7\%) y leve (2.6\%). Durante el procedimiento, el requerimiento de medicamentos inotrópicos fue del 34.6\%, de los cuales se utilizó milrinone en el 35.8\%, 19.7\% dobutamina y $2.47 \%$ levosimendam. De otro lado, fue necesaria la utilización de óxido nítrico en el $17.3 \%$ de los pacientes y transfusión de glóbulos rojos en el 35.8\% de los casos. Los demás parámetros hemodinámicos durante el procedimiento se muestran en la tabla 3.

La incidencia de complicaciones durante el cateterismo se presenta en la tabla 4, siendo la más frecuente la crisis de hipertensión pulmonar (9\%).

El drenaje venoso anómalo, la comunicación interauricular e interventricular fueron las cardiopatías congénitas que más frecuentemente presentaron desenlaces críticos (tabla 5).
El 77.7\% de los pacientes que murieron durante la hospitalización no recibió vasodilatadores pulmonares. La clasificación riesgo anestésico según la escala ASA mostró que los pacientes con mayor riesgo anestésico presentaron mayor mortalidad en los 30 días posteriores al procedimiento, siendo el $7.5 \%$ en los ASA 3, 40.0\%

TABLA 1. Características sociodemográficas y clínicas de la población de pacientes con HTP asociada a anomalía congénita asociados a cateterismo cardíaco

\begin{tabular}{|c|c|}
\hline Variable & $\mathrm{n}(\%)$ \\
\hline \multicolumn{2}{|l|}{ Edad } \\
\hline $\begin{array}{l}0 \text { a } 1 \text { mes } \\
1 \text { mes a } 1 \text { año } \\
\text { Mayores de } 1 \text { año }\end{array}$ & $\begin{array}{r}4(4.9) \\
25(30.9) \\
52(64.2)\end{array}$ \\
\hline \multicolumn{2}{|l|}{ Género } \\
\hline $\begin{array}{l}\text { Masculino } \\
\text { Femenino }\end{array}$ & $\begin{array}{l}41(51.0) \\
40(49.0)\end{array}$ \\
\hline \multicolumn{2}{|l|}{ Tipo de afiliación al SGSSS } \\
\hline $\begin{array}{l}\text { Contributivo } \\
\text { Subsidiado }\end{array}$ & $\begin{array}{l}17(17.3) \\
67(82.7)\end{array}$ \\
\hline \multicolumn{2}{|l|}{ Prematurez } \\
\hline $\begin{array}{l}\mathrm{Si} \\
\text { No }\end{array}$ & $\begin{array}{l}10(12.3) \\
71(87.7)\end{array}$ \\
\hline \multicolumn{2}{|l|}{ ASA } \\
\hline $\begin{array}{l}1 \\
2 \\
3 \\
4 \\
5\end{array}$ & $\begin{array}{r}0 \\
17(21.0) \\
53(65.4) \\
10(12.3) \\
1(1.2)\end{array}$ \\
\hline \multicolumn{2}{|l|}{ Cianosis } \\
\hline $\begin{array}{l}\mathrm{Si} \\
\mathrm{No}\end{array}$ & $\begin{array}{l}47(58.0) \\
34(42.0)\end{array}$ \\
\hline \multicolumn{2}{|l|}{ Insuficiencia renal crónica } \\
\hline $\begin{array}{l}\mathrm{Si} \\
\mathrm{No}\end{array}$ & $\begin{array}{r}3(3.7) \\
78(96.3)\end{array}$ \\
\hline \multicolumn{2}{|l|}{ Uso de Medicamentos } \\
\hline $\begin{array}{l}\text { Dilatadores pulmonares } \\
\text { IECA } \\
\text { Diuréticos } \\
\text { Ventilación mecánica previa }\end{array}$ & $\begin{array}{r}33(40.7) \\
45(55.5) \\
42(52.0) \\
9(11.1)\end{array}$ \\
\hline
\end{tabular}

Los datos son presentados como n(\%) o promedio(DS). SGSSS: Sistema de Seguridad Social en Salud. 
en los ASA 4 y 100\% en los ASA 5. No obstante, se debe recalcar que solo un paciente fue clasificado como ASA 5 previo al cateterismo. De otro lado, se encontró que del total de pacientes que murieron en el postoperatorio $(<30$ días), 2 (22.2\%) tuvieron el antecedente de prematurez.

TABLA 2. Tipo de anomalía congénita en pacientes con hipertensión pulmonar llevados a cateterismo cardíaco

\begin{tabular}{|lr|}
\hline Tipo de anomalía congénita & n (\%) \\
\hline Comunicación interauricular & $19(23.5)$ \\
\hline Comunicación interventricular & $20(25.0)$ \\
\hline Ductus arterioso persistente & $3(4.0)$ \\
\hline Doble tracto de salida del ventrículo derecho* & $6(7.4)$ \\
\hline Truncus arterioso & $3(4.0)$ \\
\hline Drenaje venoso anómalo & $11(13.7)$ \\
\hline Canal auriculoventricular & $9(11.0)$ \\
\hline Transposición de grandes vasos & $1(1.2)$ \\
\hline Coartación de aorta & $1(1.2)$ \\
\hline Sindrome de cimitarra & $2(2.5)$ \\
\hline Hipoplasia ventricular & $2(2.5)$ \\
\hline Foramen oval permeable & $3(4.0)$ \\
\hline
\end{tabular}

*(por sus siglas en inglés, RTSVD)

TABLA 3. Parámetros hemodinámicos intraoperatorios en pacientes con anomalía congénita asociada a hipertensión pulmonar llevados a cateterismo cardíaco

\section{Parámetro}

$\begin{array}{lr}\text { SatvO }_{2} & 71.0 \pm 10.3 \\ \begin{array}{l}\text { Presiones de arteria pulmonar medidas } \\ \text { en cateterismo }\end{array} & \\ \text { PSAP } & 42.5 \pm 21.7 \\ \text { PDAP } & 21.5 \pm 13.8 \\ \text { PMAP } & 30.0 \pm 15.9 \\ \text { Presión sistólica pulmonar sistémica } & 13(16.1) \\ \text { Presión sistólica pulmonar suprasistémica } & 8(10.0)\end{array}$

*Los datos son presentados como n (\%) o promedio (DS), PSAP: Presión sistólica de arteria pulmonar, PDAP: Presión diastólica de arteria pulmonar, PMAP: Presión media de arteria pulmonar
El 16.1\% y $10.0 \%$ de los pacientes presentaron durante el cateterismo mediciones de la presión sistólica pulmonar sistémica y suprasistémica, respectivamente. De estos, dos pacientes (28.6\%) presentaron crisis de hipertensión pulmonar intraoperatoria.

\section{Discusión}

Son pocos los centros especializados en el país que realizan procedimientos diagnósticos, en salas de hemodinamia como el cateterismo cardiaco en población pediátrica con hipertensión pulmonar y cardiopatía congénita, por consiguiente, no hay reporte de literatura local en relación a la incidencia de complicaciones, ya que las conductas de manejo se basan en guías del adulto o en opiniones de expertos (5). Un estudio observacional realizado en el hospital de la Universidad de Columbia Medical Center reportó complicaciones mayores (arritmias y trauma vascular) en el $1.2 \%$ de los pacientes con una mortalidad de $0.2 \%$ para cateterismo cardíaco asociado a hipertensión pulmonar con y sin anomalía congénita asociada (6). El presente trabajo contribuye al conocimiento en torno a la presentación de desenlaces críticos para el cateterismo, con un énfasis especial en el punto de vista anestésico.

TABLA 4. Prsentación de desenlaces críticos intra y posoperatorios en pacientes con anomalía congénita asociada a hipertensión pulmonar llevados a cateterismo cardíaco
Evento

Intraoperatorio

Crisis de hipertensión pulmonar

Choque cardiogénico

Hipoxemia intraoperatoria

Arritmias colapsantes

Cirugía urgente

Muerte intraoperatorio

Postoperatorio

Ventilación mecánica prolongada

Estancia prolongada uci

Muerte 30 días pos cateterismo
$7(9)$
0

$22(27)$

9 (11) 
TABLA 5. Frecuencia de desenlaces críticos de acuerdo al tipo de anomalía congénita en pacientes con hipertensión arterial llevados a cateterismo diagnóstico

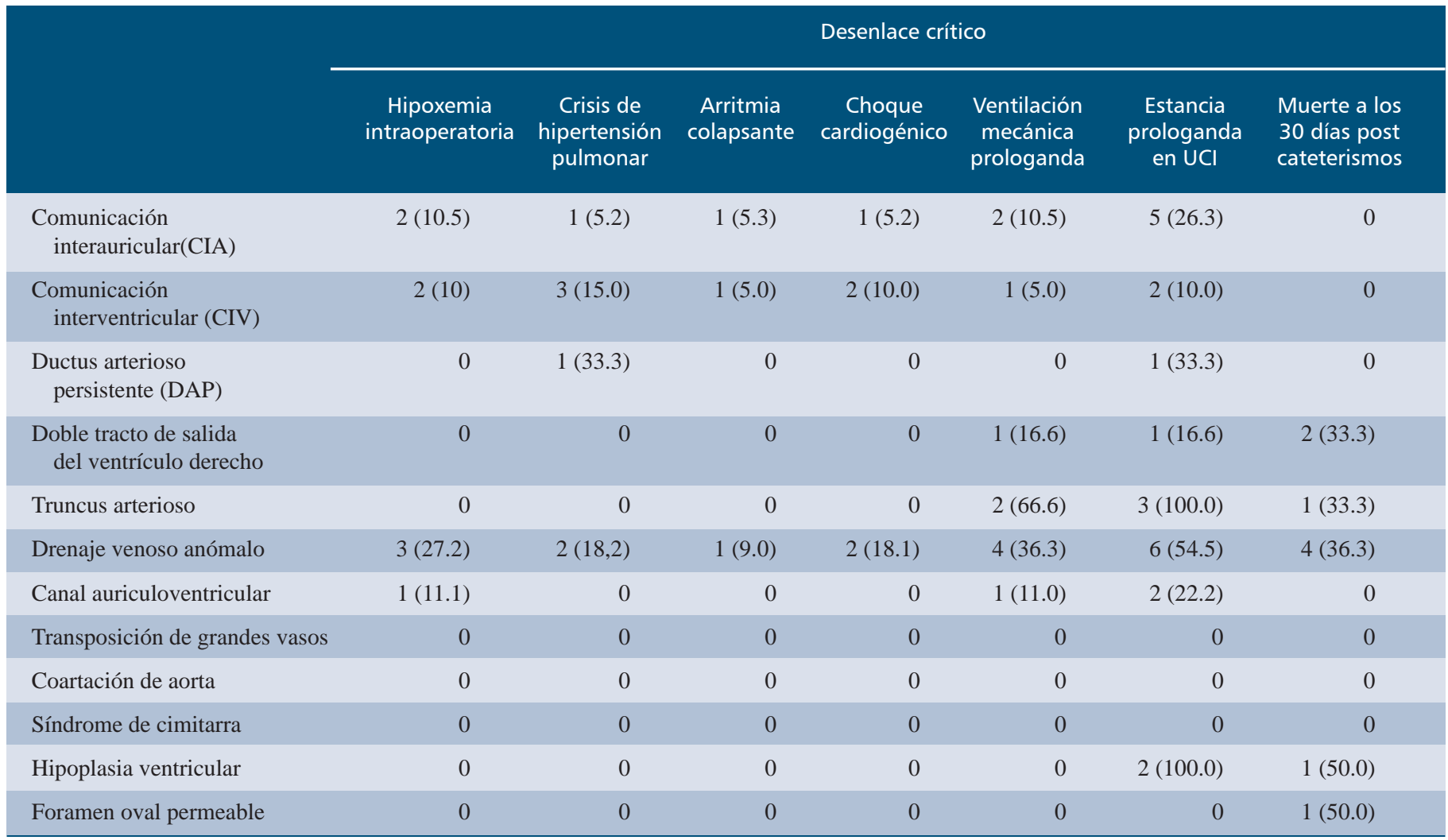

Es bien conocido que el hiperflujo pulmonar contribuye al desarrollo de un mayor grado de severidad de hipertensión pulmonar, derivando en un proceso fisiopatológico que puede llevar a hipoxemia intraoperatoria, favoreciendo el riesgo de presentar crisis de hipertensión pulmonar así mismo arritmias colapsantes, choque cardiogénico y eventualmente la muerte. En el presente estudio, la frecuencia de hipertensión pulmonar severa fue del 73.7\% y el 8\% presentaron crisis de hipertensión pulmonar, adicionalmente, el $16.1 \%$ y el $10 \%$ de los pacientes presentaron presión sistólica pulmonar sistémica y supra sistémico, respectivamente. El estudio de Carmosino et. al 2007 realizado por medio de una cohorte retrospectiva en pacientes con y sin presencia de anomalía congénita sometidos a cateterismos cardíaco identificó que los pacientes con hipertensión pulmonar en rangos sistémicos o supra-sistémicos se asociaron con mayor frecuencia a la presencia de complicaciones mayores (paro cardiaco, arritmias y muerte)(7); lo cual contrasta con los hallazgos aquí reportados donde ningún paciente falleció durante el procedimiento. De otro lado, la asociación de algún tipo de anomalía congénita con hipertensión pulmonar ha sido relacionado a un mayor riesgo anestésico durante el cateterismo cardíaco (2). Todos los anteriores factores de riesgo (HTP severa, anomalía congénita, alteraciones en los parámetros hemodinámicos) proveen un efecto que al final puede ser aditivo, considerando un alto riesgo general de eventos críticos durante la anestesia en la población de estudio (8).

Llama la atención que los pacientes que no recibieron tratamiento con vasodilatadores pulmonares, presentaron mayor frecuencia de mortalidad (77\% vs $23 \%$ ) durante los 30 días posterior al cateterismo. Los vasodilatadores pulmonares específicos como el sildenafil y bonsentan son aprobados por la FDA en pacientes pediátricos a partir de los resultados de ensayos clínicos controlados aleatorizados en donde se demostró la eficacia en la mejoría clínica, parámetros hemodinámicos medidos durante el cateterismo cardiaco y la capacidad funcional del pacien- 
te que recibía la terapia, sin embargo, no hay datos concluyentes acerca de disminución de la mortalidad $(9,10)$. De otro lado, el estudio STARTS-2 reportó mayor riesgo de mortalidad en pacientes que recibieron dosis altas de sildenafil comparados con dosis bajas cuando fueron administrados en pacientes pediátricos con HTP con diferentes tipos de cardiopatías asociadas (11). Esta situación podría estar asociada a la dificultad que existe en Colombia para acceder la atención especializada, retrasando el inicio de la terapéutica, circunstancia que podría permitir que empeore la situación fisiopatológica del paciente y que a futuro podría empeorar su pronóstico.

La principal complicación que se presentó en el estudio fue la crisis de hipertensión pulmonar (9\%), no obstante, no se presentaron muertes intraoperatorias; resultados que están acordes a la evidencia de otros estudios no restringidos a pacientes con anomalías congénitas donde la mortalidad oscila entre 0.3 y $0.8 \%$ (2). E contraste, la mortalidad en la población analizada fue considerablemente mayor en el período postquirúrgico alcanzando un valor de $11 \%$ durante los 30 días posteriores al cateterismo; este valor tal elevado podría tener relación con alta complejidad que en general representan las patologías congénitas, el compromiso del estado basal de paciente, las múltiples comorbilidades y su condición sociocultural que podría impactar negativamente en la detección y atención temprana en este grupo de pacientes.

Dado que el planteamiento del estudio no fue analítico, no se realizaron asociaciones estadísticas con respecto a la mortalidad u otros eventos críticos. En parte, por el relativamente bajo número de pacientes incluidos durante el período analizado. Por consiguiente, a futuro es posible adelanta estudios prospectivos con un mayor nivel de evidencia para probar hipótesis que surgen a partir de este trabajo. Dentro de las ventajas del presente estudio se encontró que caracteriza de forma novedosa y desde el punto de vista anestésico a la población de estudio.

Los pacientes con cardiopatía congénita e HTP sometidos a cateterismo cardíaco son pacientes de alto riesgo anestésico; la frecuencia de complicaciones intraoperatorias es alta, por lo que el anestesiólogo debe hacer un énfasis especial en la previsión del riesgo. A futuro se requiere del desarrollo de trabajos analíticos y la generación de scores de riesgo anestésico específicos para este grupo poblacional que permitan cuantificar el riesgo.
CONFLICTOS DE INTERÉS: los autores declaran que no tiene ningún conflicto de interés.

AGRADECIMIENTOS: Dra. Lina González. Asesora metodológica. Fundación Universitaria de Ciencias de la Salud. Dra. Luz Helena Arbeláez. Servicio de Cardiología Pediátrica Hospital cardiovascular del Niño de Cundinamarca. Dr. Eduardo Vanegas. Cardiología Pediatrica Hospital cardiovascular del Niño de Cundinamarca.

\section{Referencias}

1. Zarante I, Franco L, López C, Fernández N. Frecuencia de malformaciones congénitas: evaluación y pronóstico de 52.744 nacimientos en tres ciudades colombianas. Biomédica. 2010;30:65-71.

2. O’Byrne ML, Glatz AC, Hanna BD, Shinohara RT, Gillespie MJ, Dori Y, et al. Predictors of Catastrophic Adverse Outcomes in Children With Pulmonary Hypertension Undergoing Cardiac Catheterization: A Multi-Institutional Analysis From the Pediatric Health Information Systems Database. J Am Coll Cardiol. 2015;66(11):1261-9.

3. Abman SH, Hansmann G, Archer SL, Ivy DD, Adatia I, Chung WK, et al. Pediatric Pulmonary Hypertension: Guidelines From the American Heart Association and American Thoracic Society. Circulation. 2015;132(21):2037-99.

4. Arévalo-Salas Luis Alexis S-FL, Begoña Segura-Stanford, Vizcaíno-Alarcón Alfredo. Outcomes and complications of cardiac catheterization in neonates. Bol Med Hosp Infant Mex. 2008;65:126-34.

5. Vorhies EE, Ivy DD. Drug treatment of pulmonary hypertension in children. Paediatr Drugs. 2014;16(1):43-65.

6. Zuckerman WA, Turner ME, Kerstein J, Torres A, Vincent JA, Krishnan U, et al. Safety of cardiac catheterization at a center specializing in the care of patients with pulmonary arterial hypertension. Pulm Circ. 2013;3(4):831-9.

7. Carmosino MJ, Friesen RH, Doran A, Ivy DD. Perioperative complications in children with pulmonary hypertension undergoing noncardiac surgery or cardiac catheterization. Anesth Analg. 2007;104(3):521-7.

8. Stanger P, Heymann MA, Tarnoff H, Hoffman JI, Rudolph AM. Complications of cardiac catheterization of neonates, infants, and children. A three-year study. Circulation. 1974;50(3):595-608.

9. Karatza AA, Bush A, Magee AG. Safety and efficacy of Sildenafil therapy in children with pulmonary hypertension. Int J Cardiol. 2005;100(2):267-73.

10. van Loon RL, Hoendermis ES, Duffels MG, Vonk-Noordegraaf A, Mulder BJ, Hillege HL, et al. Long-term effect of bosentan in adults versus children with pulmonary arterial hypertension associated with systemic-to-pulmonary shunt: does the beneficial effect persist? Am Heart J. 2007;154(4):776-82.

11. Magee AG, Makhecha S, Bentley S. Risk-benefit considerations when prescribing phosphodiesterase-5 inhibitors in children. Expert Opin Drug Saf. 2015;14(5):633-42. 